\title{
SUBMANIFOLDS WITH PARALLEL MEAN CURVATURE VECTOR IN A SPHERE
}

\author{
MeI-Jiao Wang AND SHI-JIE LI
}

\begin{abstract}
In this paper, we study submanifolds in a unit sphere with parallel mean curvature vector, a formula of Simons' type is obtained and a corresponding pinching theorem is proved.
\end{abstract}

\section{Introduction}

Let $M$ be a closed $n$-dimensional Riemannian manifold immersed in the unit sphere $S^{n+p}$ of dimension $n+p$. Denote by $S$ the square of the length of the second fundamental form and by $H$ the mean curvature of $M$. When $M$ is minimal, J. Simons [9] obtained a pinching constant $n /\left(2-p^{-1}\right)$ of $S$ and Chern, do Carmo and Kobayashi [5] proved that if $S \leq n /\left(2-p^{-1}\right)$ on $M$, then either $M$ is totally geodesic, or the equality holds and $M$ is either a Clifford hypersurface or a Veronese surface in $S^{4}$. Then A. M. Li and J. M. Li [7] obtained a better pinching constant $(2 / 3) n$ of $S$ and proved that if $S \leq(2 / 3) n$ on $M$, then $M$ is either totally geodesic or a Veronese surface in $S^{4}$. When $M$ has parallel mean curvature vector, Z. H. Hou [6] obtained a pinching constant $2 \sqrt{n-1}$ of $S$ for the case of $p=1$, i.e., $M$ is a hypersurface of constant mean curvature immersed in the unit sphere, and characterized all such hypersurfaces with $S \leq 2 \sqrt{n-1}$. On the other hand G. Chen and X. Zou [4] discussed the case of $p>2$ and proved that if $2 \leq n \leq 7$ and $S \leq(2 / 3) n$ on $M$, then $M$ is totally umbilical.

In this paper, we prove the following:

THEOREM 1. Let $M$ be a closed n-dimensinal Riemannian manifold immersed in the unit sphere $S^{n+p}$ of dimension $n+p, p \geq 2$. If the mean curvature vector of $M$ is non-zero parallel, then

$$
\int_{M}(a S-n)\left(S-n H^{2}\right) * 1 \geq 0
$$

where $a=\max \{3 / 2, n / 2 \sqrt{n-1}\}$ and $* 1$ denotes the volume element of $M$.

The authors are supported by PNSF (Project 960179) of Guangong Province, Chına, and NSFC (Project 19771039).

Recelved February 10, 1998; revised March 23, 1998. 
THEOREM 2. Let $M$ be a closed n-dimensinal Riemannian manifold immersed in the unit sphere $S^{n+p}$ of dimension $n+p, p \geq 2$, with non-zero parallel mean curvature vector. If $S \leq n / a$ on $M$, where $a=\max \{3 / 2, n / 2 \sqrt{n-1}\}$, then $M$ is one of the following:

(1) $M$ is totally umbilical, and is a small sphere in $S^{n+1}$ with constant curvature $\left(1+|H|^{2}\right)$.

(2) $M$ is a hypersurface in $S^{n+1}$ of $S^{n+p}$, and is either $S^{n}\left(r_{0}\right)$ or $S^{1}(r) \times S^{n-1}(s)$, where $r_{0}^{2}=n /(n+2 \sqrt{n-1}), r^{2}=1 /(1+\sqrt{n-1})$ and $s^{2}=\sqrt{n-1} /(1+\sqrt{n-1})$.

\section{Preliminaries}

Let $M$ be a closed $n$-dimensional Riemannian manifold immersed in the unit sphere $S^{n+p}$ of dimension $n+p$. Denote by $A$ the Weingarten map of $M$. Choose a local orthornormal frame $\left\{e_{1}, \ldots, e_{n+p}\right\}$ in $S^{n+p}$ such that, restricted to $M,\left\{e_{1}, \ldots, e_{n}\right\}$ are tangent to $M$ and $e_{n+1}$ is in the direction of the mean curvature vector of $M$ in $S^{n+p}$, i.e., the normalized mean curvature vector. Then we have that $\operatorname{tr} A_{n+1}=n H, \operatorname{tr} A_{\alpha}=0$, for $n+2 \leq \alpha \leq n+p$, and $S=|A|^{2}=$ $\sum_{\alpha=n+1}^{n+p}\left|A_{\alpha}\right|^{2}=\sum_{\alpha=n+1}^{n+p} \operatorname{tr} A_{\alpha}^{2}$, where $A_{\alpha}=A_{e_{\alpha}}$ denotes the Weingarten map with respect to $e_{\alpha}$.

As Alencar and do Carmo in [1] and Santos in [8], we define a bilinear map $\Phi: T M \times T M \rightarrow T^{\perp} M$ by

$$
\Phi(X, Y)=\sum_{\alpha=n+1}^{n+p}\left\langle\Phi_{\alpha} X, Y\right\rangle e_{\alpha},
$$

where $\Phi_{\alpha}$ is given by

$$
\left\{\begin{aligned}
\Phi_{n+1} & =H \text { id }-A_{n+1}, \\
\Phi_{\alpha} & =-A_{\alpha}, \quad n+2 \leq \alpha \leq n+p .
\end{aligned}\right.
$$

Then we have

$$
|\Phi|^{2}:=\sum_{\alpha=n+1}^{n+p} \operatorname{tr} \Phi_{\alpha}^{2}=S-n H^{2} .
$$

We need the following results of W. Santos [8], and A. M. Li and J. M. Li [7].

LEMMA 1 [8]. Let $B_{1}$ and $B_{2}$ be symmetric $(n \times n)$-matrices such that $\left[B_{1}, B_{2}\right]=0$ and $\operatorname{tr} B_{1}=\operatorname{tr} B_{2}=0$. Then

$$
\operatorname{tr} B_{1}^{2} B_{2} \leq \frac{n-2}{\sqrt{n(n-1)}}\left(\operatorname{tr} B_{1}^{2}\right) \sqrt{\operatorname{tr} B_{2}^{2}} .
$$

We rewrite Theorem 1 of [7] as follows. 
Lemma 2 [7]. Let $B_{1}, \ldots, B_{p}, p \geq 2$, be symmitric $(n \times n)$-matrices. Then

$$
\sum_{\alpha, \beta=1}^{p}\left\{\operatorname{tr}\left[B_{\alpha}, B_{\beta}\right]^{2}-\left(\operatorname{tr} B_{\alpha} B_{\beta}\right)^{2}\right\} \geq-\frac{3}{2}\left(\sum_{\alpha=1}^{p} \operatorname{tr} B_{\alpha}^{2}\right)^{2} .
$$

We also need the following results of B. Y. Chen and K. Yano [3] and Z. H. Hou [6].

Proposition 1 [3]. Let $M^{n}$ be a non-minimal pseudo-umbilical submanifold of $S^{n+p}$. If the mean curvature vector of $M$ is parallel, then $M$ is a minimal submanifold of a hypersphere of $S^{n+p}$.

Proposition 2 [6]. Let $M^{n}$ be a closed hypersurface of constant mean curvature in $S^{n+1}$. Then

(1) If $S<2 \sqrt{n-1}, M$ is a small hypersphere $S^{n}(r)$ of radius $r=\sqrt{n /(n+S)}$.

(2) If $S=2 \sqrt{n-1}, M$ is either $S^{n}\left(r_{0}\right)$ or $S^{1}(r) \times S^{n-1}(s)$, where $r_{0}^{2}=$ $n /(n+2 \sqrt{n-1}), r^{2}=1 /(1+\sqrt{n-1})$ and $s^{2}=\sqrt{n-1} /(1+\sqrt{n-1})$.

\section{Proof of theorems}

First, we estimate $\Delta|\Phi|^{2}$. Santos [8] calculated the Laplacian $\Delta|\Phi|$ of $\Phi$ by using a formula of $\mathrm{J}$. J. Erbacher for $\Delta|A|^{2}$ and obtained that if the mean curvature vector of $M$ is parallel, with the above notation, we have

$$
\begin{aligned}
\frac{1}{2} \Delta|\Phi|^{2}= & \sum_{\alpha=n+1}^{n+p}\left|\nabla \Phi_{\alpha}\right|^{2}+n\left(1+H^{2}\right)|\Phi|^{2}-n H \sum_{\alpha=n+1}^{n+p} \operatorname{tr} \Phi_{n+1} \Phi_{\alpha}^{2} \\
& -\sum_{\alpha, \beta=n+1}^{n+p}\left(\operatorname{tr} \Phi_{\alpha} \Phi_{\beta}\right)^{2}+\sum_{\alpha, \beta>n+1}^{n+p} \operatorname{tr}\left(\left[\Phi_{\alpha}, \Phi_{\beta}\right]\right)^{2}
\end{aligned}
$$

where $\left[\Phi_{\alpha}, \Phi_{\beta}\right]=\Phi_{\alpha} \Phi_{\beta}-\Phi_{\beta} \Phi_{\alpha}$.

Rewrite (3.1) as the following:

$$
\begin{aligned}
\frac{1}{2} \Delta|\Phi|^{2}= & \sum_{\alpha=n+1}^{n+p}\left|\nabla \Phi_{\alpha}\right|^{2}+n\left(1+H^{2}\right)|\Phi|^{2}-\left(\operatorname{tr} \Phi_{n+1}^{2}\right)^{2} \\
& -n H \sum_{\alpha=n+1}^{n+p} \operatorname{tr} \Phi_{n+1} \Phi_{\alpha}^{2}+\sum_{\alpha, \beta>n+1}^{n+p}\left\{\operatorname{tr}\left(\left[\Phi_{\alpha}, \Phi_{\beta}\right]\right)^{2}-\left(\operatorname{tr} \Phi_{\alpha} \Phi_{\beta}\right)^{2}\right\} \\
& -2 \sum_{\alpha>n+1}^{n+p}\left(\operatorname{tr} \Phi_{n+1} \Phi_{\alpha}\right)^{2}
\end{aligned}
$$

Since $\operatorname{tr} \Phi_{\alpha}=0, \quad \alpha=n+1, \ldots, n+p, \quad$ and $\quad\left[\Phi_{n+1}, \Phi_{\alpha}\right]=\left[A_{n+1}, A_{\alpha}\right]=0$, $\alpha=n+2, \ldots, n+p$, we may apply Lemma 1 to the fourth term of (3.2) and 
have

$$
\begin{aligned}
\sum_{\alpha=n+1}^{n+p} \operatorname{tr} \Phi_{n+1} \Phi_{\alpha}^{2} & \leq \frac{n-2}{\sqrt{n(n-1)}}\left(\sum_{\alpha=n+1}^{n+p} \operatorname{tr} \Phi_{\alpha}^{2}\right) \sqrt{\operatorname{tr} \Phi_{n+1}^{2}} \\
& =\frac{n-2}{\sqrt{n(n-1)}}|\Phi|^{2}\left|\Phi_{n+1}\right| .
\end{aligned}
$$

Applying Lemma 2 to the fifth term of (3.2), we may have for $p \geq 3$

$$
\begin{aligned}
\sum_{\alpha, \beta>n+1}^{n+p}\left\{\operatorname{tr}\left(\left[\Phi_{\alpha}, \Phi_{\beta}\right]\right)^{2}-\left(\operatorname{tr} \Phi_{\alpha} \Phi_{\beta}\right)^{2}\right\} & \geq-\frac{3}{2}\left(\sum_{\alpha>n+1}^{n+p}\left|\Phi_{\alpha}\right|^{2}\right)^{2} \\
& =-\frac{3}{2}\left(|\Phi|^{2}-\left|\Phi_{n+1}\right|^{2}\right)^{2} .
\end{aligned}
$$

Since when $p=2$, (3.4) becomes

$$
-\left(\operatorname{tr} \Phi_{n+2} \Phi_{n+2}\right)^{2} \geq-\frac{3}{2}\left(\operatorname{tr} \Phi_{n+2}^{2}\right)^{2}
$$

which holds of course and we really have (3.4) for $p \geq 2$.

For the last term of (3.2), Cauchy-Schwarz's inequality gives the following for any $\alpha$.

$$
\left(\operatorname{tr} \Phi_{n+1} \Phi_{\alpha}\right)^{2} \leq\left|\Phi_{n+1}\right|^{2}\left|\Phi_{\alpha}\right|^{2}
$$

Therefore, we have

$$
-2 \sum_{\alpha>n+1}^{n+p}\left(\operatorname{tr} \Phi_{n+1} \Phi_{\alpha}\right)^{2} \geq-2\left|\Phi_{n+1}\right|^{2}\left(|\Phi|^{2}-\left|\Phi_{n+1}\right|^{2}\right) .
$$

Apply (3.3)-(3.6) to (3.2), we may have for $p \geq 2$

$$
\begin{aligned}
\frac{1}{2} \Delta|\Phi|^{2} \geq & \sum_{\alpha=n+1}^{n+p}\left|\nabla \Phi_{\alpha}\right|^{2}+n\left(1+H^{2}\right)|\Phi|^{2}-\frac{n-2}{\sqrt{n(n-1)}} n H\left|\Phi_{n+1}\right||\Phi|^{2} \\
& -\frac{3}{2}\left(|\Phi|^{2}-\left|\Phi_{n+1}\right|^{2}\right)^{2}-\left|\Phi_{n+1}\right|^{4}-2\left|\Phi_{n+1}\right|^{2}\left(|\Phi|^{2}-\left|\Phi_{n+1}\right|^{2}\right) \\
= & \sum_{\alpha=n+1}^{n+p}\left|\nabla \Phi_{\alpha}\right|^{2}+\frac{1}{2}\left|\Phi_{n+1}\right|^{2}\left(|\Phi|^{2}-\left|\Phi_{n+1}\right|^{2}\right) \\
& +|\Phi|^{2}\left\{n\left(1+H^{2}\right)-\frac{(n-2) \sqrt{n}}{\sqrt{(n-1)}} H\left|\Phi_{n+1}\right|\right. \\
& \left.-\frac{3}{2}\left(|\Phi|^{2}-\left|\Phi_{n+1}\right|^{2}\right)-\left|\Phi_{n+1}\right|^{2}\right\}
\end{aligned}
$$




$$
\begin{aligned}
= & \sum_{\alpha=n+1}^{n+p}\left|\nabla \Phi_{\alpha}\right|^{2}+\frac{1}{2}\left|\Phi_{n+1}\right|^{2}\left(|\Phi|^{2}-\left|\Phi_{n+1}\right|^{2}\right) \\
& +|\Phi|^{2}\left\{n-\frac{3}{2}\left(|\Phi|^{2}-\left|\Phi_{n+1}\right|^{2}\right)\right. \\
& \left.+\left(n H^{2}-\frac{(n-2) \sqrt{n}}{\sqrt{(n-1)}} H\left|\Phi_{n+1}\right|-\left|\Phi_{n+1}\right|^{2}\right)\right\} .
\end{aligned}
$$

In the last term of (3.7), we need to estimate the following

$$
z\left(H,\left|\Phi_{n+1}\right|\right)=n H^{2}-\frac{(n-2) \sqrt{n}}{\sqrt{(n-1)}} H\left|\Phi_{n+1}\right|-\left|\Phi_{n+1}\right|^{2} .
$$

Let $x=\sqrt{n} H$ and $y=\left|\Phi_{n+1}\right|$, then (3.8) becomes

$$
z\left(H,\left|\Phi_{n+1}\right|\right)=x^{2}-\frac{n-2}{\sqrt{n-1}} x y-y^{2}, \quad y \geq 0,
$$

which could be consider as a quadratic surface in $E^{3}$. By the following coordinate transformation:

$$
\left\{\begin{array}{l}
u=\frac{1}{\sqrt{2 n}}\{(1+\sqrt{n-1}) x+(1-\sqrt{n-1}) y\} \\
v=\frac{1}{\sqrt{2 n}}\{-(1-\sqrt{n-1}) x+(1+\sqrt{n-1}) y\}
\end{array}\right.
$$

we may find that

$$
\begin{aligned}
z\left(H,\left|\Phi_{n+1}\right|\right) & =\frac{n}{2 \sqrt{n-1}}\left(u^{2}-v^{2}\right) \\
& =-\frac{n}{2 \sqrt{n-1}}\left(v^{2}+u^{2}-2 u^{2}\right) \\
& \geq-\frac{n}{2 \sqrt{n-1}}\left(v^{2}+u^{2}\right) \\
& =-\frac{n}{2 \sqrt{n-1}}\left(x^{2}+y^{2}\right) \\
& =-\frac{n}{2 \sqrt{n-1}}\left(n H^{2}+\left|\Phi_{n+1}\right|^{2}\right)
\end{aligned}
$$

Substituting (3.10) into (3.7), we have

$$
\begin{aligned}
\frac{1}{2} \Delta|\Phi|^{2} \geq & \sum_{\alpha=n+1}^{n+p}\left|\nabla \Phi_{\alpha}\right|^{2}+\frac{1}{2}\left|\Phi_{n+1}\right|^{2}\left(|\Phi|^{2}-\left|\Phi_{n+1}\right|^{2}\right) \\
& +|\Phi|^{2}\left\{n-\frac{3}{2}\left(|\Phi|^{2}-\left|\Phi_{n+1}\right|^{2}\right)-\frac{n}{2 \sqrt{n-1}}\left(n H^{2}+\left|\Phi_{n+1}\right|^{2}\right)\right\}
\end{aligned}
$$


And taking $a=\max \{3 / 2, n /(2 \sqrt{n-1})\}$, we may finally obtain the following, since $|\Phi|^{2}=S-n H^{2}$

$$
\frac{1}{2} \Delta|\Phi|^{2} \geq \sum_{\alpha=n+1}^{n+p}\left|\nabla \Phi_{\alpha}\right|^{2}+\frac{1}{2}\left|\Phi_{n+1}\right|^{2}\left(|\Phi|^{2}-\left|\Phi_{n+1}\right|^{2}\right)+|\Phi|^{2}(n-a S) .
$$

Proof of Theorem 1. Integrating (3.12) on $M$, we get (1.1). q.e.d.

Proof of Theorem 2. If $S \leq n / a$ on $M$, then either $S \equiv n H^{2}$ or $S \equiv n / a$ by Theorem 1. In the first case, $M$ is totally umbilical and thus contained in $S^{n+1}$ with constant curvature $\left(1+|H|^{2}\right)$. (cf. [2, pp. 49-50]).

For the later, we may see from (3.12) that both $\left|\Phi_{n+1}\right|^{2}\left(|\Phi|^{2}-\left|\Phi_{n+1}\right|^{2}\right)$ and $\sum_{\alpha=n+1}^{n+p}\left|\nabla \Phi_{\alpha}\right|^{2}$ must vanish.

(1) If $\left|\Phi_{n+1}\right|=0$, which implies that $\left|A_{n+1}\right|^{2}=n H^{2}, M$ is a pseudo-umbilical submanifold of $S^{n+p}$. Applying Proposition 1 of Chen and Yano, we find that $M$ is minimal in a hypersphere $S^{n+p-1}$ of $S^{n+p}$ with constant scalar curvature. But, since the equality in (3.10) holds when $s=n / a$, we may find that $u$ must vanish. Consequently, from (3.9) the mean curvature $H$ of $M$ would be vanish which contradicts the hypothesis of the theorem.

(2) If $|\Phi|^{2}-\left|\Phi_{n+1}\right|^{2}=0$, and plus that $\nabla \Phi_{\alpha}=0$, for $\alpha=n+1, \ldots, n+p$, we may find that $M$ is a hypersurface of $S^{n+1}$ in $S^{n+p}$. Then by Proposition 2 of Hou, we obtain that

(a) For $2 \leq n \leq 7$, we have $a=3 / 2$, and then $S=2 n / 3<2 \sqrt{n-1}$, thus $M$ is a small hypersphere $S^{n}(r)$ of radius $r=\sqrt{n /(n+S)}$.

(b) For $n \geq 8$, we have $a=n / 2 \sqrt{n-1}$, and then $S=2 \sqrt{n-1}$, thus $M$ is either $S^{n}\left(r_{0}\right)$ or $S^{1}(r) \times S^{n-1}(s)$, where $r_{0}^{2}=n /(n+2 \sqrt{n-1}), r^{2}=1 /(1+\sqrt{n-1})$ and $s^{2}=\sqrt{n-1} /(1+\sqrt{n-1})$.

q.e.d.

Remark. When the dimension $n$ of $M$ is in $2 \leq n \leq 7$, then $a=3 / 2$ and our Theorem 1 is a generalization of Theorem 2 of A. M. Li and J. M. Li [7].

Theorem 2 generalizies Theorem 2 of [4].

\section{REFERENCES}

[1] H. Alencar AND M. Do Carmo, Hypersurfaces with constant mean curvature in spheres, Proc. Amer. Math. Soc., 120 (1994), 1223-1229.

[2] B. Y. ChEN, Geometry of Submanifolds, M. Dekker, New York, 1973.

[3] B. Y. ChEN AND K. YANO, Minımal submanifolds of a higher dimensional sphere, Tensor (N. S.), 22 (1971), 369-373.

[ 4 ] G. Chen AND X. Zou, Rigidity of compact submanifolds in a unit sphere, Koda1 Math. J., 18 (1995), 75-85.

[ 5 ] S. S. Chern, M. Do CARMo AND S. Kobayashi, Minımal submanifolds of a sphere with second fundamental form of constant length, Functional Analysis and Related Fields (Proc. Conf. for Stone), Springer-Verlag, New York, 1970, 59-75. 
[6] Z. H. Hou, Hypersurfaces in a sphere with constant mean curvature, Proc. Amer. Math. Soc., 125 (1997), 1193-1196.

[7] A. M. Li AND J. M. LI, An intrnsic rigidity theorem for mınımal submanifolds in a sphere, Arch. Math. (Basel), 58 (1992), 582-594.

[8] W. SANTos, Submanifolds with parallel mean curvature vector in spheres, Tôhoku Math. J., 46 (1994), 403-415.

[9] J. Simons, Minımal varieties in Riemannian manifolds, Ann. of Math., 88 (1968), 62-105.

Department of Mathematics

Guangzhou Educational College

GUANGZHOU 510030

CHINA

DePartment OF Mathematics

SOUTH CHINA Normal UNIVERSITY

GUANGZHOU 510631

CHINA

E-mail: lisj@scnu.edu.cn 